\title{
End diastolic flow velocity just beneath the aortic isthmus assessed by pulsed Doppler echocardiography: a new predictor of the aortic regurgitant fraction
}

\author{
Christophe Tribouilloy, Pierre Avinée, Wei Feng Shen, Jean-Luc Rey, Michel Slama, \\ Jean-Philippe Lesbre
}

\begin{abstract}
End diastolic flow velocity just beneath the aortic isthmus was measured within 72 hours of cardiac catheterisation by pulsed Doppler echocardiography in 30 controls and 61 patients with aortic regurgitation. The end diastolic flow velocity was determined at the peak $R$ wave on a simultaneously recorded electrocardiogram. In all controls there was no reverse flow at the end diastole beneath the aortic isthmus. In patients with aortic regurgitation the end diastolic flow velocity correlated well with the angiographic grade of regurgitation $(\mathbf{r}=0.81)$ and regurgitant fraction $(r=0.82)$. The mean (SD) values were $6 \cdot 3(5 \cdot 2), 12 \cdot 2(4 \cdot 3), 22 \cdot 1(5 \cdot 7)$, and $34 \cdot 3(9 \cdot 3)$ $\mathrm{cm} / \mathrm{s}$ for patients with regurgitant fraction of $<20 \%$, between $20 \%$ and $40 \%$, between $41 \%$ and $60 \%$, and $>60 \%$, respectively. An end diastolic flow velocity of $>18 \mathrm{~cm} / \mathrm{s}$ predicted a regurgitant fraction of $\geqslant 40 \%$ with a sensitivity of $88.5 \%$ and a specificity of $96 \%$.

The study suggests that the pulsed Doppler derived end diastolic flow velocity is a useful index in the routine non-invasive assessment of the severity of aortic regurgitation.
\end{abstract}

With the widespread application of both pulsed and continuous wave Doppler echocardiography ${ }^{1-3}$ and colour flow imaging, ${ }^{4}$ several variables have been proposed as a measure of the severity of chronic aortic regurgitation. Clinical study showed pandiastolic flow in the descending aorta in patients with aortic regurgitation. ${ }^{5}$ We found that it was easy to measure the end diastolic flow velocity just beneath the aortic isthmus by pulsed Doppler echocardiography and that this method provides semiquantitative information on the degree of aortic regurgitation. ${ }^{6}$ In the present study we investigated further the usefulness of this measurement in estimating the aortic regurgitant fraction.

\section{Patients and methods}

PATIENTS

Seventy patients with chronic aortic regurgitation underwent cardiac catheterisation and Doppler echocardiography within 72 hours of each other. Nine patients with poor echogenicity were excluded (pure aortic regurgitation in four and associated aortic stenosis in five). We studied the remaining 61 patients (43 men and 18 women, mean (SD) age 60 (17) years (range 22-84)). All patients were in stable sinus rhythm and had no angiographically significant mitral regurgitation. Forty two patients had pure aortic regurgitation and 19 had associated aortic stenosis. The cause of aortic regurgitation was rheumatic fever in 22 , degenerative abnormality in 29 , infective endocarditis in eight, post-traumatic lesion in one, and prosthetic valve dysfunction in one. All patients had had a clinical diagnosis of aortic regurgitation for more than six months.

Thirty patients without valve disease served as a control group. Cardiac catheterisation and angiography were performed because of atypical chest pain. All patients had normal electrocardiograms, chest $x$ ray, and Doppler findings.

DOPPLER ECHOCARDIOGRAPHIC EXAMINATION We used an ATL Mark 600 or a HewlettPackard 77020 system equipped with a 3 or 2.5 $\mathrm{MHz}$ pulsed Doppler transducer respectively. Patients were placed in a supine position with the head overextended and the shoulders raised. The transducer was positioned in the suprasternal notch and directed downwards to bring the ultrasound beam on to the thoracic aorta at a point between its horizontal and descending portions. The sample volume was placed in the proximal descending aorta just beneath the aortic isthmus and the origin of the left subclavian artery. The Doppler beam was aligned in parallel with the axis of aortic blood flow by showing a maximal systolic (negative) flow velocity curve. Doppler filters were adjusted to minimal values, and gains were reduced to obtain a contrasted but uninterrupted display of the model Doppler frequencies. Patients with chronic aortic regurgitation often show reverse flow velocities throughout diastole in the descending aorta and the flow velocity is relatively constant after mid diastole. ${ }^{7}$ The end diastolic flow velocity $(\mathrm{cm} / \mathrm{s})$ was measured at the peak of the $R$ wave on a simultaneously recorded electrocardiogram (fig 1).

The reproducibility of this measurement using this method was tested in 20 patients with aortic regurgitation: the mean (SD) intra 
Figure 1 Measurement of end diastolic flow velocity by pulsed Doppler echocardiography. (A) Ultrasound beam aligned with the axis of the descending aorta with sample volume just beneath the aortic isthmus and junction with subclavian artery. (B) Flow velocity curve. Systolic flow velocity appears below the $O$ line and diastolic flow velocity above it. The end diastolic flow velocity was measured at the peak of the $R$ wave on $a$ simultaneously recorded electrocardiogram.
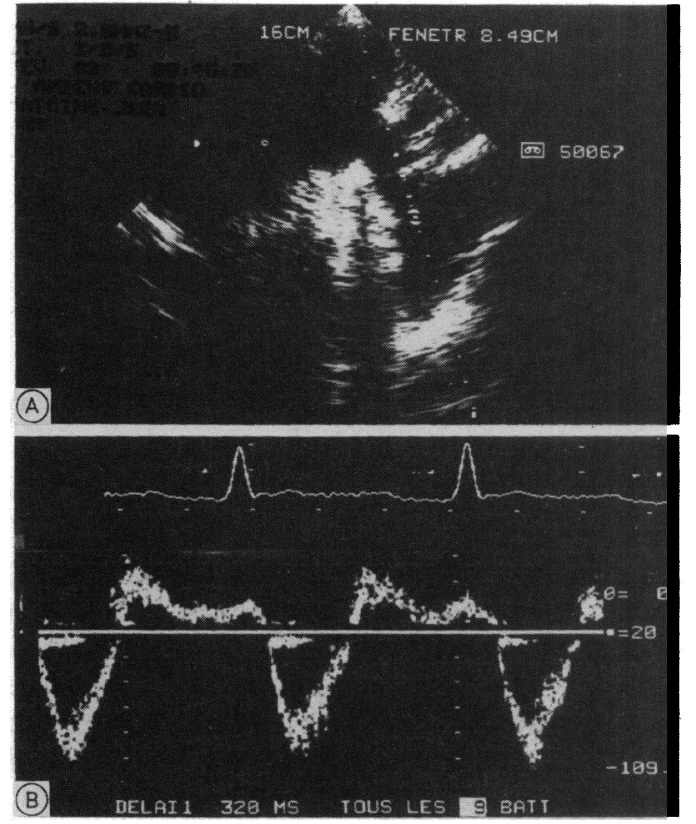

(B)

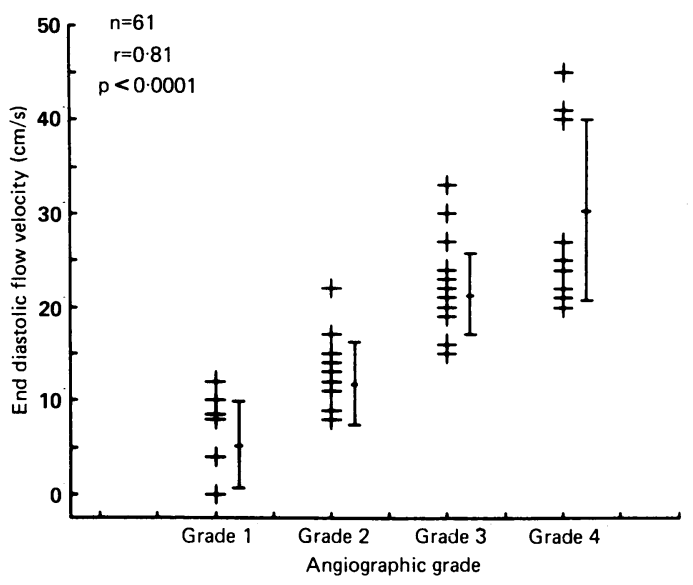

and inter observer variabilities were 4.4 $(3 \cdot 2) \%$ and $5 \cdot 6(3 \cdot 6) \%$ respectively.

\section{CARDIAC CATHETERISATION}

Right and left heart catheterisations were performed within 72 hours of the Doppler echocardiographic studies. Cardiac output was determined by the thermodilution technique and total left ventricular output was measured from left ventriculography performed in the $30^{\circ}$ right anterior oblique projection. Regurgitant fraction was calculated by the standard formula. Aortography was performed in $30^{\circ}$ right anterior oblique projection with $40-60 \mathrm{ml}$ of contrast media injected through a $7 \mathrm{~F}$ pigtail catheter posi-

Table 1 Pulsed Doppler prediction of angiographic grade of aortic regurgitation by end diastolic flow velocity. The pulsed Doppler class accorded with the angiographic class in 39 of 61 patients and no patient was misclassified by more than one class

\begin{tabular}{llccc}
\hline \multirow{4}{*}{ Angiographic grade } & \multicolumn{4}{l}{ Doppler end diastolic flow velocity $(\mathrm{cm} / \mathrm{s})$} \\
\cline { 2 - 5 } & $0-9$ & $>9-18$ & $>18-30$ & $>30$ \\
\hline I & 5 & 3 & 0 & 0 \\
II & 3 & 13 & 2 & 0 \\
III & 0 & 4 & 16 & 2 \\
IV & 0 & 0 & 8 & 5 \\
\hline
\end{tabular}

tioned $2-3 \mathrm{~cm}$ above the aortic valve. The degree of aortic regurgitation was graded on a scale of I to IV by the method of Sellers et al. ${ }^{8}$

\section{STATISTICAL ANALYSIS}

The results were expressed as mean (1 SD). We tested the relation between the end diastolic flow velocity and angiographic grades of regurgitation by the Spearman correlation analysis; for continuous data we used linear regression analysis.

\section{Results}

CONTROL GROUP

In all 30 controls the mean ( $1 \mathrm{SD}$ ) value of peak systolic flow velocity was $1 \cdot 10(0 \cdot 20) \mathrm{m} / \mathrm{s}$. Early diastolic flow beneath the aortic isthmus was detected with a velocity of $<0.25 \mathrm{~m} / \mathrm{s}$ and lasting $<200 \mathrm{~ms}$. There was no reverse flow during mid and late diastole.

AORTIC REGURGITATION

In 61 patients there was a good correlation between end diastolic flow velocity and the angiographic grade of aortic regurgitation (fig 2).

Mean (SD) end diastolic flow velocity in eight patients with grade I regurgitation was $5.3(4 \cdot 6) \mathrm{cm} / \mathrm{s}$ (end diastolic flow velocity was zero in four), for 18 patients with grade II regurgitation it was $11.8(4 \cdot 4) \mathrm{cm} / \mathrm{s}$, for 22 patients with grade III regurgitation it was $21 \cdot 4$ $(4.3) \mathrm{cm} / \mathrm{s}$, and for 13 patients with grade IV regurgitation it was $30 \cdot 4(9 \cdot 6) \mathrm{cm} / \mathrm{s}$. Though there was some overlap in end diastolic flow velocity among individuals with each of the four grades of aortic regurgitation, this measurement was a good indication of the severity of aortic regurgitation. Thirty one of 35 patients with end diastolic flow velocity $>18 \mathrm{~cm} / \mathrm{s}$ had grade III or IV regurgitation, whereas 24 of 26 patients with end diastolic flow velocity $<18 \mathrm{~cm} / \mathrm{s}$ had grade I or II. Therefore, an end diastolic flow velocity $>18 \mathrm{~cm} / \mathrm{s}$ predicted severe aortic regurgitation ( $\geqslant$ grade III) with a sensitivity of $88.5 \%$ and a specificity of $92 \cdot 3 \%$ (table 1 ).

The end diastolic flow velocity correlated

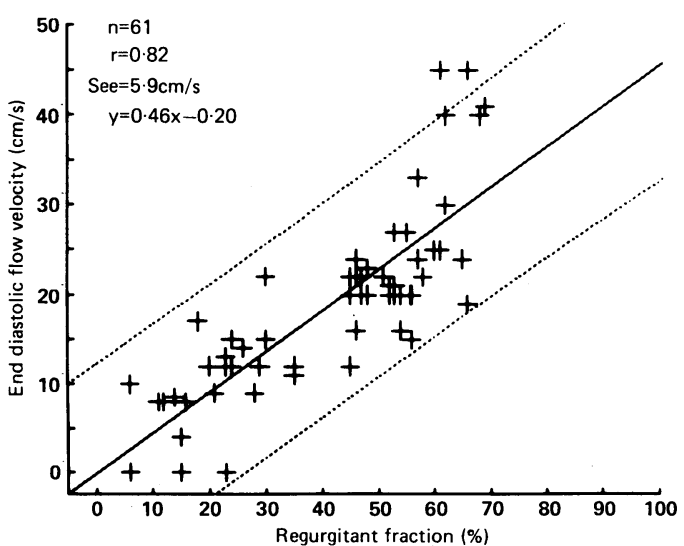

Figure 3 Correlation between end diastolic flow velocity measured by pulsed Doppler method and regurgitant fraction by haemodynamic method. Broken lines show fraction by haemodynamic method. Broken lines show
$95 \%$ confidence interval and solid line is the regression line. Some points represent more than one patient. 
Table 2 Pulsed Doppler prediction of angiographic regurgitant fraction by end diastolic flow velocity. The pulsed Doppler class accorded with the angiographic class in 46 of 61 patients and no patient was misclassified by more than one class

\begin{tabular}{llccc}
\hline & \multicolumn{4}{l}{ Doppler end diastolic flow velocity $(\mathrm{cm} / \mathrm{s})$} \\
\cline { 2 - 5 } Regurgitant fraction $(\%)$ & $0-9$ & $>9-18$ & $>18-30$ & $>30$ \\
\hline $0-20$ & 7 & 3 & 0 & 0 \\
$21-40$ & 1 & 14 & 1 & 0 \\
$41-60$ & 0 & 4 & 20 & 2 \\
$>60$ & 0 & 0 & 4 & 5 \\
\hline
\end{tabular}

well with the regurgitant fraction measured by a combined haemodynamic and angiographic method (fig 3). Mean (SD) end diastolic flow velocity was $6 \cdot 3(5 \cdot 2) \mathrm{cm} / \mathrm{s}$ in 10 patients with a regurgitant fraction $<20 \%, 12 \cdot 2(4.3) \mathrm{cm} / \mathrm{s}$ in 16 patients with a regurgitant fraction between $20-40 \%, 22 \cdot 1(5 \cdot 7) \mathrm{cm} / \mathrm{s}$ in 26 patients with a regurgitant fraction between $41-60 \%$, and $34 \cdot 3$ $(9.3) \mathrm{cm} / \mathrm{s}$ in nine patients with a regurgitant fraction $>60 \%$. The end diastolic flow velocity reliably classified the regurgitant fraction. Thirty one of 35 patients with end diastolic flow velocity $>18 \mathrm{~cm} / \mathrm{s}$ had a regurgitant fraction $>40 \%$. In contrast, 25 of 26 patients with end diastolic flow velocity $<18 \mathrm{~cm} / \mathrm{s}$ had a regurgitant fraction of $<40 \%$.Therefore, an end diastolic flow velocity of $>18 \mathrm{~cm} / \mathrm{s}$ identified patients with regurgitant fraction $>40 \%$ with a sensitivity of $88.5 \%$ and a specificity of $96 \%$ (table 2).

\section{Discussion}

An excellent alignment can be achieved between the ultrasound beam and blood flow during Doppler assessment of flow velocity in the descending aorta and the flow velocity at the end of diastole measured just beneath the aortic isthmus is a good indicator of the severity of aortic regurgitation.

Previous studies of the flow profiles in the aorta showed an irregular flow velocity pattern in the ascending aorta with moderate or severe regurgitation. ${ }^{10}$ Because flow velocity across the lumen varies in diastole calculation of forward or reverse flow is unreliable. In contrast, flow in the descending aorta produces a much flatter velocity profile. ${ }^{9-11}$ In most of our patients we found a narrow band of frequencies even when regurgitation was severe. Furthermore, movement during the cardiac cycle is much less in the aortic isthmus than the ascending aorta, ${ }^{11}$ allowing better positioning of the sample volume. In our controls the early diastolic reverse flow detected in the descending aorta by the Doppler method may reflect compliance of the aortic wall, and it may be impossible to define a shape that takes account of the alteration in compliance and the diastolic reflux caused by aortic regurgitation. ${ }^{10}$ For these reasons, flow velocity during late diastole in the descending aorta has been investigated extensively to assess the severity of aortic regurgitation.

Boughner used continuous Doppler echocardiography to measure the flow velocity in the descending aorta; he found that the ratio between the diastolic and systolic flow velocitytime integrals allowed measurement of aortic regurgitant fraction. ${ }^{5}$ Touche et al proposed a modified method using pulsed Doppler in which the flow velocity-time integral ratio was multiplied by the ratio of aortic diastolic area to systolic area obtained from an $M$ mode echocardiogram. ${ }^{11}$ They also reported a good correlation between this measurement and regurgitant fraction determined invasively. Diebold $e t$ al also reported that the ratio of the end diastolic to the peak systolic velocity can also be obtained from pulsed Doppler echocardiography and that it gives semiquantitative information about the severity of aortic regurgitation. ${ }^{10}$ Pulsed Doppler is better than continuous wave Doppler because it is not affected by the venous flow velocities during diastole because the site of the sample volume can be accurately located. These three methods of measuring the regurgitant fraction depend largely on determinations of systolic flow velocity. Thus they may not be reliable in the presence of associated aortic stenosis. ${ }^{10}$

A preliminary study from our laboratory showed that a simple measure of the end diastolic flow velocity gave an accurate semiquantitative measure of the severity of aortic regurgitation, and it can be easily determined in most patients with aortic regurgitation. ${ }^{6}$ The results of this study confirm our previous observations and further indicate the high sensitivity and specificity of an end diastolic flow velocity of $>18 \mathrm{~cm} / \mathrm{s}$ in predicting moderate to severe (grade $\geqslant$ III) aortic regurgitation and identified patients with a regurgitant fraction of $>40 \%$. This suggests that the end diastolic flow velocity may also be used to predict the aortic regurgitant fraction.

There are several potential limitations of this method. If aortic regurgitation is slight, velocities in the reverse flow will be low and may not be recorded if a filter with too high a cut off frequency is used. In patients with a ductus arteriosus, aortopulmonary fistula, aortic coarctation at the isthmus level, or aortic dissection, this method is not feasible because of a continuous flow component in these regions. ${ }^{11}$ Because the accuracy of end diastolic flow velocity in measuring the severity of aortic regurgitation relies on a relatively constant reverse flow velocity throughout diastole, the regurgitant fraction may be overestimated during tachycardia ( $>120$ beats/min) and underestimated during bradycardia ( $<50$ beats) min). ${ }^{12}$ In atrial fibrillation, when the diastole is prolonged, there may be no end diastolic flow. The compliance of the left ventricle in acute aortic regurgitation might well be quite different from that of patients with chronic aortic regurgitation. There may be equilibration of aortic and ventricular pressures before end diastole, which may lead to cessation of reverse flow before the proposed measurement.

The end diastolic flow velocity just beneath the aortic isthmus can be measured easily in most patients with chronic aortic regurgitation and is an accurate indicator of the regurgitant fraction. It may be useful in the routine assessment of patients with chronic aortic regurgita- 
tion. The effect of various afterloads on this relation should be investigated.

1 Ciobanu M, Abbasi AS, Allen M, Herner A, Spellberg R Pulsed Doppler echocardiography in the diagnosis and
estimation of severity of aortic insufficiency. Am J Cardiol 1982;49:339-43.

2 Masuyama T, Kodama K, Kitabatake A, et al. Non invasive evaluation of aortic regurgitation by continuous wave Doppler echocardiography. Circulation 1986;73:460-6.

3 Teague S, Heinsimer J, Anderson J, et al. Quantification of aortic regurgitation utilizing continuous wave Doppler ultrasound. J Am Coll Cardiol 1986;3:592-9.

4 Perry GJ, Helmcke F, Nanda NC, Byard C, Soto B. Evaluation of aortic insufficiency by Doppler color flow mapping. J Am Coll Cardiol 1987;9:952-9.

5 Boughner DR. Assessment of aortic insufficiency by transcutaneous Doppler ultrasound. Circulation 1975;52. 874-80.

6 Lesbre JP, Avinee P, Isorni C, Rey JL, Choquet D, Mertl C Echo Doppler assessment of aortic insufficiency by a new pulsed index [Abstract]. Eur Heart $J$ 1988;9(suppl I): $\mathrm{I}-42$. 7 Hatle L, Angelsen B. Doppler ultrasound in cardiology: Philadelphia; Lea and Febiger, 1985:97-292.

8 Sellers RD, Levy MJ, Amplatz K, Lillehei CW. Left retrograde cardioangiography in acquired cardiac disease: technic, indications and interpretations in 700 cases. $\mathrm{Am} \mathrm{J}$ Cardiol 1964;14:437-47.

9 Schultz DL. Pressure and flow in large arteries. In: Bergel $\mathrm{DH}$, ed. Cardiovascular fluid dynamics. London: DH, ed. Cardiovascular f

10 Diebold B, Peronneau P, Blanchard D, et al. Non-invasive quantification of aortic regurgitation by Doppler echocardiography. Br Heart $J$ 1983;49:167-73.

11 Touche T, Prasquier R, Nitenberg A, De Zuterre D, Gourgon R. Assessment and follow up of patients with aortic regurgitation by an updated Doppler echocardiographic measurement of the regurgitant fraction in the aortic arch. Circulation 1985;72:819-24.

12 Avinee P, Rey JL, Isorni C, Darras B, Lesbre JP. Validité comparée des critères de quantification de l'insuffisance aortique en doppler pulsé et continu. Arch Mal Coeur 1988;81:895-901. 